\title{
Dynamic reduction in the calculation of bridge structures
}

\author{
Aleksander Makarov ${ }^{1}$, Sergey Kalinovsky ${ }^{2 *}$, and Natalia Ermilova ${ }^{3}$ \\ ${ }^{1}$ Volgograd State Technical University, Construction and operation of transport facilities \\ Department, 400074 Volgograd, Russian Federation \\ ${ }^{2}$ Volgograd State Technical University, Hydraulic and earth structures Department, 400074 \\ Volgograd, Russian Federation \\ ${ }^{3}$ Volgograd State Technical University, Engineering Graphics, Standardization and Metrology, \\ 400074 Volgograd, Russian Federation
}

\begin{abstract}
In modern bridge construction, on the one hand, there are increasing trends towards increasing bridge spans, which requires reducing the weight of structures. On the other hand, the use of structural elements made of various composite materials is expanding, which allows to significantly reduce the weight of the bridge structures as a whole. However, the creation of new forms of span structures of bridges requires more detailed calculations in order to optimize such forms, in particular the role of calculating the dynamic impact, because with increasing spans and weight loss, increases design flexibility and sensitivity to dynamic loads. In the present paper, the problems of solving an incomplete algebraic problem of eigenvalues and eigenvectors are considered. To increase the accuracy of the calculation and exclude the use of high-order matrices, a method of sequential reduction of the stiffness and equivalent mass matrices is proposed. The method is based on the construction of partial systems using a static transformation, followed by the solution of its own problem for the partial system. In the process of solving this problem through the eigenvectors of the system, the minor unknowns are reduced to the main ones. Dynamic reduction showed high calculation accuracy..
\end{abstract}

\section{Introduction}

Currently, a large number of bridges are built according to individual projects. Moreover, both unique structures and quite ordinary ones are created [1,2]. Modern technologies allow us to use an extensive list of materials in bridge construction: aluminum, steels of various strengths-bimetallic [3], carbon fiber and other composites [4,5,6]. The use of new materials, in contrast to standard structures, can significantly increase the efficiency of construction, requires more detailed calculations for strength, stability and, in particular, for dynamic impacts.

Despite the above-mentioned opportunities in the use of a wide range of materials and technologies, practice shows that designers in their work use well-established ideas about

\footnotetext{
* Corresponding author: sk0522@yandex.com
} 
the work of bridge structures, without seeking to find and apply the optimal parameters of superstructures. This was noted earlier in the works where the spans of girder bridges [3,7] and arch bridges [8] are considered. Many questions arise, in particular, when analyzing the stress-strain state of a metal superstructure with an orthotropic plate (Fig. 1). For example, deflections of the covering sheet cause longitudinal cracks in the asphalt concrete pavement of the bridge bed. The problem is now most often solved by increasing the thickness of the coating, which increases the weight of the structure and the stress in the longitudinal edge of the orthotropic plate adjacent to the main beam of the superstructure. Therefore, such a design solution is not effective and requires a detailed comprehensive study to find other solutions.

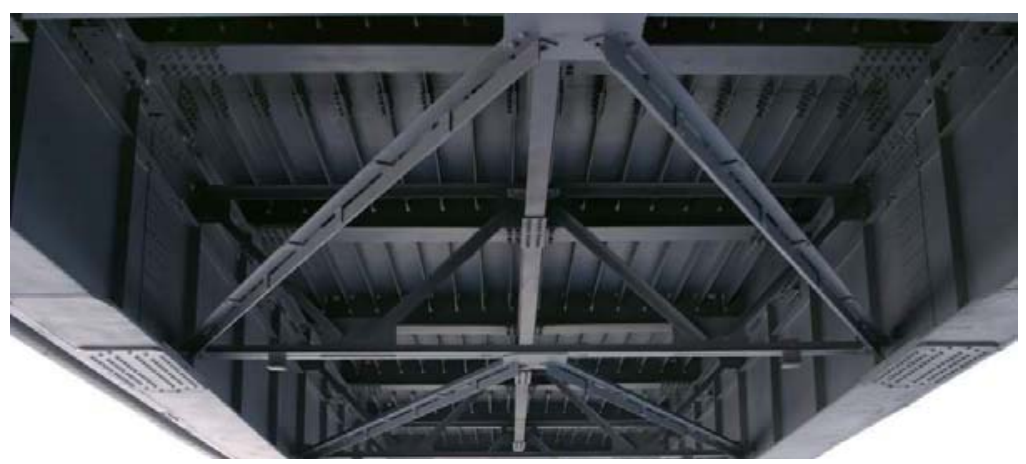

Fig. 1. Superstructure with orthotropic plate.

The pronounced tendency to increase the length of bridge spans requires a reduction in the weight of the spans, which leads to an increase in the flexibility of the structure. This means that the superstructure becomes more sensitive to dynamic loads. In the history of bridge construction, there are many examples of sensitivity to aerodynamic loads. Fluctuations of bridge spans were also observed more than once, and cases of their collapse in a very short time. To avoid the appearance of resonant oscillations or a significant decrease in their amplitudes, calculations for dynamic effects are necessary, which are carried out by software complexes based on the finite element method.

\section{Justification of the dynamic reduction technique}

The design scheme of a metal or bimetallic bridge superstructure with an orthotropic plate is a complex continuum-rod system. To ensure sufficient accuracy of the dynamic calculation, a detailed discretization of the structure is necessary - splitting it into finite elements of the smallest possible size. This leads to the construction of high-order matrices $\left(N=10^{6}-10^{8}\right)$, which are formed and solved during the calculation process. The large size of the matrices allows you to significantly increase the accuracy of calculations, but it takes up a large amount of memory and significantly increases the calculation time. Therefore, the task is to reduce the order of matrices in RAM.

The solution of the dynamic problem without taking into account damping [9] in the form of $M Y^{\prime \prime}+R Y=-M Y_{0}^{\prime \prime}$ reduces to solving the incomplete problem of eigenvalues and eigenvectors. The system of solving equations in the form of the displacement method has the form:

$$
[K-\lambda M]\{y\}=0
$$

It should be noted that it makes practical sense to find not the entire spectrum of oscillation frequencies, but only the lower part of the spectrum, because the actual driving 
loads acting on the superstructure cannot have a high-frequency range. Therefore, it is possible to lower the order of the initial stiffness and mass matrices by developing reduction techniques. The problem of solving partial algebraic problem of eigenvalues and eigenvectors are addressed and solved from a different angle and is implemented as in the deflection method [10], and in the form of mixed-method [11], and using mathematical modeling $[12,13]$. A known technique of static reduction, which attracts simplicity, but is not accurate because it does not take into account the dynamic properties of the system [13, $14,15,16]$.

\section{The main stages of solving the problem and their analysis}

The proposed method takes into account the dynamic properties of the system and, due to the vastness of the matrices, is performed not simultaneously, but sequentially in blocks. The main degrees of freedom of the $-r$ system are chosen in such a way that the array of secondary degrees of freedom $-s$ is divided into separate blocks that have no connection with each other. This will allow for reduction without loss of interaction links.

Reduction of the first block of secondary degrees of freedom.

The Gaian transform matrix of order $(r+s) \times N$, has the form

$$
A_{s r}^{(1)}=\left[\begin{array}{l}
E_{r+s} \\
-\left[K_{s s}^{(2)}\right]^{-1} K_{s r}^{(2)} \\
-\left[K_{s s}^{(3)}\right]^{-1} K_{s r}^{(3)} \\
\cdots \cdots \cdots \cdots \cdots \cdots \\
-\left[K_{s s}^{t}\right]^{-1} K_{s r}^{(t)}
\end{array}\right]
$$

and is used to obtain the matrices of the first partial system-the block of the main and the first block of the subordinate degrees of freedom of the order $(\mathrm{r}+\mathrm{s})$

$$
\begin{gathered}
K^{*}=\left[A_{s r}^{(1)}\right]^{T} \cdot K \cdot A_{s r}^{(1)} \\
M^{*}=\left[A_{s r}^{(1)}\right]^{T} \cdot M \cdot A_{s r}^{(1)} .
\end{gathered}
$$

When solving their own task, may to find the own vectors

$$
\left[K^{*}-\lambda M^{*}\right]\{y\}=0
$$

Then the first subordinate block is dynamically reduced to the main block. The relation between the main and subordinate unknowns for each own vectors and for the first vectors of the system is expressed by the equality

$$
\left[Y_{s r}\right]=A_{s r} \cdot\left[Y_{r r}\right]
$$

where $Y_{r r}$ - matrix of the main components of the first $r$ eigenvectors; $Y_{s r}$ - matrix of subordinate components of the same vectors. Then the matrix converter will have the form:

$$
A_{s r}=\left[Y_{s r}\right]\left[Y_{s r}\right]^{-1}
$$

and the expression for the own vector is written as follows:

$$
\{y\}=\left\{\begin{array}{l}
y_{r} \\
y_{s}
\end{array}\right\}=\left[\begin{array}{c}
E_{r} \\
A_{s r}
\end{array}\right]=B\left\{y_{r}\right\}
$$

The matrices of stiffness and equivalent masses of the main degrees of freedom, taking into account the influence of the first subordinate block, have the form

$$
K_{r r}^{(1)}=B^{T} K^{*} B
$$




$$
M_{r r}^{(1)}=B^{T} M^{*} B
$$

Similarly, the reduction of all subordinate blocks to the block of main variables is performed by repeating the transformations (2) - (7). The stiffness and mass matrices of a completely reduced system are obtained by summing all reduced partial systems minus the matrices of the main block $K_{r r}, M_{r r}$, the influence of which was taken into account at each stage of reduction.

$$
\begin{aligned}
\widetilde{K}_{r r} & =\sum_{i=1}^{t} K_{r r}^{(i)}-(t-1) K_{r r} \\
\widetilde{M}_{r r} & =\sum_{i=1}^{t} M_{r r}^{(i)}-(t-1) M_{r r}
\end{aligned}
$$

This dynamic reduction algorithm shows a good result of solving its own problem in the lower part of the oscillation frequency spectrum, but it has a technical drawback. Expressions (2) - (3)use high-order matrices that occupy a large amount of RAM. We propose a different way of forming the stiffness and mass matrices of partial systems without using the original high-order matrices entirely.

First, we obtain the reduced stiffness and mass matrices of the main block using successive static transformations $[18,19,20]$.

$$
\begin{gathered}
\bar{K}_{r r}=K_{r r}-\sum_{i=1}^{t} K_{r s}^{(i)} A_{s r}^{(i)} \\
\bar{M}_{r r}=M_{r r}-\sum_{i=1}^{t} M_{r s}^{(i)} A_{s r}^{(i)}-\sum_{i=1}^{t}\left[A_{s r}^{(i)}\right]^{T} M_{s r}^{(i)}+\sum_{i=1}^{t}\left[A_{s r}^{(i)}\right]^{T} M_{s s}^{(i)} A_{s r}^{(i)}
\end{gathered}
$$

here $A_{s r}^{(i)}=\left[K_{s s}^{(i)}\right]^{T} K_{s r}^{(i)}$ static Guyane transformation for the $i$-th subordinate block.

The stiffness and mass matrices of the first partial system in this case will have the form

$$
\begin{gathered}
K^{*}=\left[\begin{array}{l}
\hat{K}_{r r} K_{r s}^{1} \\
K_{s r}^{1} K_{s s}^{1}
\end{array}\right] \\
M^{*}=\left[\begin{array}{c}
\hat{M}_{r r} M_{r s}^{1} \\
M_{s r}^{1} M_{s s}^{1}
\end{array}\right]
\end{gathered}
$$

where $K_{s s}^{1} \quad M_{s s}^{1}$ stiffness and mass matrices of the first subordinate block; $\hat{K}_{r r} \hat{M}_{r r}$ matrices of the block of main unknowns after static reduction to them of all subordinate blocks, except the first, which can be obtained in accordance with expressions (8) using the inverse transformation:

$$
\begin{gathered}
\hat{K}_{r r}=\bar{K}_{r r}+K_{r s}^{1} A_{s r}^{1} \\
\hat{M}_{r r}=\bar{M}_{r r}+M_{r s}^{1} A_{s r}^{1}+\left[M_{r s}^{1} A_{s r}^{1}\right]^{T}-\left[A_{s r}^{1}\right]^{T} M_{s s}^{1} A_{s r}^{1}
\end{gathered}
$$

Next, dynamic reduction is performed in accordance with the algorithm of expressions (4) - (7). According to the same scheme, the matrices of the following partial systems are constructed and their reduction to the main block of variables is performed. The final matrices are obtained according to the expressions (8)

We illustrate the proposed reduction method by the example of a beam that is pinched at the ends and has 142 dynamic degrees of freedom. For simplicity, the length of the finite element is assumed to be unit $l=1$. The bending stiffness is also $E I=1$. The reduction is carried out to 22 degrees of freedom. We will choose the main degrees of freedom so that the subordinate ones are divided into six independent blocks with the number of unknowns of twenty in each. Thus, the initial data of the example has the form: $N=142, r=22, t=6, s$ $=20$. To analyze the effectiveness of the proposed method, three calculations were performed: the complete problem of eigenvalues and eigenvectors was solved, the partial 
problem of own values and own vectors with static matrix reduction was solved, and the partial problem of eigenvalues and eigenvectors with sequential dynamic reduction was solved.

The results of calculations of the own values of the main variables are presented in Table 1.

Table 1. Results of calculations of the own values of the main variables.

\begin{tabular}{|c|c|c|c|c|c|}
\hline \multirow{3}{*}{$\begin{array}{l}\text { Number } \\
\text { of the } \\
\text { own } \\
\text { value }\end{array}$} & \multicolumn{5}{|c|}{ The own values obtained of the solution } \\
\hline & \multirow[t]{2}{*}{$\begin{array}{c}\text { complete } \\
\text { problem } \\
\mathrm{N}=142 \\
\lambda\end{array}$} & \multicolumn{2}{|c|}{$\begin{array}{l}\text { static reduction of the } \\
\text { system to } 22 \text { degrees } \\
\text { of freedom }\end{array}$} & \multicolumn{2}{|c|}{$\begin{array}{l}\text { dynamic reduction of the system. The } \\
\text { main block has } 22 \text { degrees of freedom, } \\
\text { the subordinate blocks have } 6 \text { blocks of } \\
20 \text { degrees of freedom }\end{array}$} \\
\hline & & $\lambda$ & $\Delta, \%$ & $\lambda$ & $\Delta, \%$ \\
\hline 1 & 2 & 3 & 4 & 5 & 6 \\
\hline 1 & 0.0000186 & 0.0000186 & 0 & 0.0000186 & 0 \\
\hline 2 & 0.000141 & 0.000141 & 0.03 & 0.000141 & 0 \\
\hline 3 & 0.000543 & 0.000543 & 0.1 & 0.000543 & 0 \\
\hline 4 & 0.00148 & 0.00149 & 0.26 & 0.00148 & 0 \\
\hline 5 & 0.00331 & 0.00333 & 0.55 & 0.00331 & 0 \\
\hline 6 & 0.00647 & 0.00653 & 1.04 & 0.00647 & 0 \\
\hline 7 & 0.0114 & 0.0116 & 1.75 & 0.0114 & 0 \\
\hline 8 & 0.0189 & 0.0194 & 2.68 & 0.0189 & 0 \\
\hline 9 & 0.0295 & 0.0306 & 3.77 & 0.0295 & 0 \\
\hline 10 & 0.044 & 0.0462 & 4.67 & 0.044 & 0 \\
\hline 11 & 0.0634 & 0.0655 & 3.26 & 0.0634 & 0 \\
\hline 12 & 0.0885 & 0.107 & 17.68 & 0.0885 & 0 \\
\hline 13 & 0.12 & 0.145 & 17.23 & 0.12 & 0 \\
\hline 14 & 0.16 & 0.199 & 19.49 & 0.16 & 0 \\
\hline 15 & 0.209 & 0.27 & 22.68 & 0.209 & 0 \\
\hline 16 & 0.268 & 0.366 & 26.58 & 0.268 & 0.01 \\
\hline 17 & 0.34 & 0.493 & 31.07 & 0.34 & 0.03 \\
\hline 18 & 0.424 & 0.662 & 35.97 & 0.426 & 0.05 \\
\hline 19 & 0.524 & 0.883 & 40.67 & 0.528 & 0.44 \\
\hline 20 & 0.64 & 1.159 & 44.73 & 0.65 & 0.84 \\
\hline 21 & 0.775 & 1.468 & 47.20 & 0.847 & 8.56 \\
\hline 22 & 0.93 & 1.751 & 46.91 & 1.083 & 14.16 \\
\hline
\end{tabular}

\section{Conclusions}

Based on the above, the following main conclusions can be drawn:

1. The proposed new design and planning solutions for bridge structures need detailed calculations in order to identify the most effective forms.

2. The proposed method for determining the frequencies of free vibrations of bridges does not use high-order matrices, spends less machine time and allows you to get only the necessary data for analyzing the dynamic behavior of the structure.

3. The proposed method of sequential dynamic reduction showed high calculation accuracy for $90 \%$ of the calculated values, compared to $36 \%$ calculated according to the known method. 


\section{References}

1. I.I. Ovchinnikov, A. B. Karakhanyan, I. G. Ovchinnikov, Yu. P. Skachkov, Modern pedestrian and bicycle bridges (basic design concepts and examples). 140p. (Penza State University of Architecture and Construction, Penza, 2018)

2. N.V. Ivanova, A.V. Makarov, S.A. Kalinovsky, The main aspects of the bionic bridges building, IOP Conference Series: Materials Science and Engineering, 698(7) 077007 (2019) 7p. URL: https://iopscience.iop.org/article/10.1088/1757-899X/698/7/077007

3. A.V. Makarov, S.A.Kalinovsky, Design features of bimetallic bridges, in Proceedings of the XXII International Scientific Conference «Construction the Formation of Living Environment» (FORM-2019). E3S Web of Conferences 97, 06001 (2019) 9p. https://doi.org/10.1051/e3sconf/20199706001

4. O.N. Stolyarov, V.Y. Olshevskiy, A.E. Dontsova, Y.A. Demidova, Carbon fibers in bridge construction. Construction of Unique Buildings and Structures 3(78) (2019) pp. 36-49. DOI: 10.18720/CUBS.78.3

5. A.G. Strugach, A.G. Trifonov, Architecture of contemporary pedestrian bridges made of fiber-reinforced composite materials. Russian journal of transport engineering [online] 1(6) (2019). DOI: 10.15862/17SATS119 (http://dx.doi.org/10.15862/17SATS119)

6. A.E. Ushakov, U.G. Klenin, T.G. Sorina, A.H. Khairetdinov, A.A. Safonov, Bridge structures made of composites. Composites and nanostructures 3 (2009) URL: https://www.researchgate.net/publication/310601955_Mostovye_konstrukcii_iz_komp ozitov

7. A.V. Makarov, S.A. Kalinovsky, Economical reinforced concrete overpass. Graphical method of calculationg, IOP Conference Series: Materials Science and Engineering, 962(2) 022051 (2020) 7p. URL: https://iopscience.iop.org/article/10.1088/1757899X/962/2/022051/pdf. DOI: 10.1088/1757-899X/962/2/022051

8. A.V. Makarov, S.A. Kalinovsky, Methods of regulating thrust in design of arch bridges, IOP Conference Series: Materials Science and Engineering, 451(1) 012054 (2018) 7p. URL: https://iopscience.iop.org/article/10.1088/1757899X/451/1/012054/pdf. DOI: 10.1088/1757-899X/451/1/012054

9. I. M. Babakov, Theory Of Oscillations (Drofa Publishing House, Moscow 2004) 591 p.

10. V. A. Ignatiev, V. N. Romashkin, Algebraic problem of eigenvector and eigenvalues of high order in tasks of dynamics and stability of a construction. Internet-Vestnik VolgGASU 2(38) (2015). URL: http://vestnik.vgasu.ru/attachments/7IgnatievRomashkin-2015_2_38_.pdf

11. A.V. Ignatiev, V. A. Ignatiev, E. A. Gamzatova, Analysis of flexible plates having rigid inclusions or holes according to finite element method in the form of a classical mixed method. Izvestiya vysshego uchebnykh zavedeniy. Construction. 9 (2017) pp. 514

12. A. V. Ignatiev, V. A. Ignatiev, Mathematical modeling of an incomplete algebraic problem of eigenvalues and eigenvectors for obtaining a reduced frequency equation and solving it, VII International Symposium «Actual problems of computer modeling of structures and structures (APCSCE 2018)» (Russian Academy of Architecture and Construction Sciences, Novosibirsk State University of Architecture and Construction. - Novosibirsk, 2018) p. 263

13. V. A. Ignatiev, A. V. Makarov, Solution of the incomplete algebraic problem of eigenvectors and eigenvalues for dynamics and stability problems by the method of 
frequency-dynamic condensation, Construction mechanics and calculation of structures 1 (2005) pp. 14-20

14. R. Gallagher, The finite element method. The basics. (Translated from English - Mir, Moscow, 1984) $428 \mathrm{p}$

15. O. Zenkevich, K. Morgan, Finite elements and approximation (Translated from English - Mir, Moscow, 1986) 318 p

16. S.S. Gusev, B. A. Kuranov, Features of the application of the superelement method in problems of stability and free oscillations. Proceedings of the 14th All-Union Conference on the theory of plates and shells. (Kutaisi. October 20-23, 1987) Vol. 1 (Publishing House of the Tbilisi University, Tbilisi, 1987) pp. 445-449

17. A. S. Gorodetsky, The possibilities of using superelements in solving various problems of construction mechanics. Construction mechanics and calculation of structures 4 (2015) pp. 51-56

18. V. A. Ignatiev, A. V. Makarov, K. A. Sukhin, Calculation of rod systems based on the energy variant of the method of frequency-dynamic condensation using the eigenvectors of subsystems. In the collection: Urban agglomerations in landslide areas. Materials of the International Scientific Conference. (VSUASE,Volgograd, 2003) pp. 113-120

19. V. A. Ignatiev, V. N. Romashkin, Using spline-collocation interpolation to find the reduced frequency spectrum and forms of free oscillations of systems with a large number of degrees of freedom, in proceedings of the V International Symposium " Actual problems of computer modeling of buildings and structures» (ISU, Irkutsk, 2014) pp. 104-105

20. O. L. Rudykh Improving the method of reduced elements on the basis of synthesis with the method of superelements, Izvestiya vuzov. Construction 7 (2006) pp. 103-108. 